University of Warwick institutional repository: http://go.warwick.ac.uk/wrap This paper is made available online in accordance with publisher policies. Please scroll down to view the document itself. Please refer to the repository record for this item and our policy information available from the repository home page for further information.

To see the final version of this paper please visit the publisher's website. Access to the published version may require a subscription.

Author(s): Vousden, Janet; Maylor, Elizabeth

Article Title: Speech errors across the lifespan

Year of publication: 2006

Link to published version: http://dx.doi.org/

10.1080/01690960400001838

Publisher statement:None 


\title{
Running head: SPEECH ERRORS ACROSS THE LIFESPAN
}

Speech Errors Across the Lifespan

\author{
Janet I. Vousden and Elizabeth A. Maylor \\ University of Warwick, Coventry
}

Address:

Department of Psychology

University of Warwick

Coventry CV4 7AL

UK

Tel: +44 (0)2476 522611

email: J.I.Vousden@warwick.ac.uk 


\begin{abstract}
Dell, Burger and Svec (1997) proposed that the proportion of speech errors classified as anticipations (e.g., “moot and mouth”) can be predicted solely from the overall error rate, such that the greater the error rate, the lower the anticipatory proportion (AP) of errors. We report a study examining whether this effect applies to changes in error rates that occur developmentally and as a result of aging. Speech errors were elicited from 8- and 11-year-old children, young adults and older adults. The error rate decreased and the AP increased from children to young adults, but neither error rate nor AP differed significantly between young and older adults. In cases where fast speech resulted in a higher error rate than slow speech, the AP was lower. Thus, there was overall support for Dell et al's prediction from speech error data across the lifespan.
\end{abstract}




\section{Speech Errors Across the Lifespan}

Over the decades, many models of language production have been informed by the patterns of speech errors that emerge when the language production system malfunctions. This method has been a popular way of gaining insight into the mechanisms that underpin error-free production. One area where speech error data have been particularly useful is the understanding of the mechanism that effects the serial order of phonemes during speech production. A complete theory should account for data gathered from a wide range of ages, from a developing child to older adults. However, while considerable speech error data have been collected from young adults (e.g., Boomer \& Laver, 1968; Dell, 1984, 1986, 1989, 1990; Dell, Burger, \& Svec, 1997; Dell \& Reich, 1981; Fromkin, 1971; Garnham, Shillcock, Brown, Mill, \& Cutler, 1982; Garrett, 1975, 1976; Harley, 1984, Harley \& MacAndrew, 1995; MacKay, 1970; Nooteboom, 1969, 1980; Schwartz, Saffran, Bloch, \& Dell, 1994; Shattuck-Hufnagel, 1979, 1987; Shattuck-Hufnagel \& Klatt, 1979; Stemberger, 1982, 1985, 1990), there are few data from children (Jaeger, 1992; Stemberger, 1989; Warren, 1986; Wijnen, 1992), and even fewer from older adults (James, 2000; McKay \& James, in press). The types of speech error data that inform theories of serial order in speech are contextual substitution errors (e.g., "moot and mouth" instead of "foot and mouth"). Some theories based primarily on such data have made predictions concerning how the patterns of these speech errors may change across the lifespan. In this article we test predictions made by Dell et al. (1997) by examining patterns of experimentally induced speech errors in young children, young adults and older adults. 
We begin by briefly outlining the types of speech errors that inform theories of serial order in speech and go on to describe predictions for age-related effects from a model developed by Dell et al. (1997). We also consider predictions derived from a more recent model of serial order in speech (Vousden, Brown, \& Harley, 2000). Two experiments are then presented that examine how the proportions of different speech error types change from young children through adulthood to older adults. Such data have not previously been available to evaluate how particular age-related changes could be mediated; this article therefore provides a starting point for examining age-related effects in speech production.

\section{Serial Order and Speech Errors}

Order is important to distinguish between the production of different words that are composed of the same phonemes, e.g., tap, pat, and apt. A mechanism to effect the correct serial order of phonemes during speech production is therefore crucial to ensure the correct words are spoken. One of the simplest mechanisms of serial order is achieved via a left-to-right chain of associative bonds between phonemes (e.g., Wickelgren, 1969). However, a mechanism for sequencing must be more sophisticated than a sequence of associations between adjacent elements. The types of speech errors people produce and the manner in which such errors appear to be constrained is problematic for such a mechanism (see e.g., Burgess \& Hitch, 1992; Henson, Norris, Page, \& Baddeley, 1996; Houghton, 1990; Houghton \& Hartley, 1996; Lashley, 1951). For example, when phoneme errors occur, phonemes almost always interact with other phonemes in the same syllabic position (Garrett, 1975; MacKay, 1970; Shattuck-Hufnagel, 1979; Vousden et al., 2000). Phonemes also tend to interact with nearby rather than distant phonemes when 
an error occurs (e.g., Broeke \& Goldstein, 1980; Garcia-Albea, del Viso, \& Igoa, 1989; Vousden et al., 2000). These errors cannot be explained by a mechanism in which serial order is maintained by following the chain of associations from each phoneme to the next. Any model of serial order in phonological encoding must therefore address the observed constraints. One way to gain insight into how a mechanism for serial order might work, therefore, is to look at how errors are constrained when the ordering of phonemes during production goes awry. Contextual errors (largely anticipations, perseverations, and exchanges) reflect cases where the correct phonemes are spoken but their order is incorrect in some way. Anticipations occur when a phoneme that appears later in an utterance is spoken too early, e.g., "moot and mouth" instead of "foot and mouth”. Perseverations occur when a phoneme that appears early in an utterance is spoken later, e.g., “foot and fouth” instead of “foot and mouth”. Anticipations are thought to reflect the pre-activation of upcoming phonemes in the utterance whereas perseverations are thought to reflect the persisting activation of past phonemes. Factors Affecting Past vs. Future Activation

One measure of the relative level of activation focused on the past versus the future is the Anticipatory Proportion (AP) of errors (Schwartz et al., 1994). The AP is calculated as the proportion of all anticipation and perseveration errors that are anticipatory, i.e. AP = anticipations / (anticipations + perseverations). Previous findings have shown that the AP varies in different populations and under different conditions. For example, Schwartz et al. (1994) noted that an aphasic speaker made more perseverations than anticipations. His AP was 0.32 , which turned out to be reliably lower than that of nonaphasic speakers, who have an AP of 0.75 (as determined by an analysis 
of the Garnham et al., 1982, corpus of spontaneously occurring speech errors). There is also some evidence that young children have a lower AP than adults: Stemberger (1989) noted that the errors of two young children contained more perseverations than anticipations, while adults tended to anticipate more than perseverate.

Several experimental manipulations of adults’ speech point to a more robust variation in the AP. When participants were asked to repeat tongue twisters many times, Schwartz et al. (1994) and Dell et al. (1997) both found that practice had a significant effect on the AP. When participants first started to repeat tongue twisters, not only did they make many errors, but a large proportion of them were perseverations. However, with practice their error rate fell and errors were mainly anticipatory. This is the anticipatory practice effect. The AP also varies systematically when the time available to speak is varied. When phrases are repeated either at a slow rate or at a long deadline in comparison to a fast rate or short deadline, perseverations are less likely than anticipations (Dell, 1990; Dell et al., 1997). This is the anticipatory speech-rate effect. These variations in AP led Schwartz et al. (1994) to conclude that, in general, the AP is higher when the overall error rate is lower. Schwartz et al. characterised "good" error patterns as those having low error rates and a high AP, whereas "bad" error patterns show elevated error rates and a low AP. Several recent models of serial order in speech production (Dell et al., 1997; Vousden et al., 2000) have sought to explain how and under what conditions these errors prevail. Dell et al. proposed a mathematical model relating various factors that appear to affect the production of contextual errors. Vousden et al. on the other hand proposed a model of how the serial order mechanism might function and why contextual errors occur. 


\section{Modelling "Good" and "Bad” Error Patterns}

Dell et al. (1997) developed a general frame-based model motivated by characteristics common to several prominent models of serial order in speech production (e.g., Dell, 1986; Houghton, 1990; MacKay, 1987; Rumelhart \& Norman, 1982). The model contains four nodes: the plan node connects to content nodes (which belong to different structural categories) for the past, present and future, with a long-term positive weight, w. A structural frame controls serial order by sending signals to the content nodes: 0 is sent to all members of the "past" category, 1.0 is sent to all members of the "present" category, and $b$ is sent to all members of the "future" category (where $b$ is a positive fraction representing priming). These signals combine multiplicatively with inputs from the plan node, in effect temporarily altering the net weights so that the activation to the past becomes 0 (effectively inhibiting it), the present becomes 1.0 (activating it), and the future becomes $b w$ (priming it). The perseveratory tendency of the model is determined by the ratio of activation of the past to present nodes; likewise, the anticipatory tendency is determined by the ratio of activation of the future to present nodes. Activation levels for each of the past, present, and future nodes depend on the model parameters, which reflect knowledge of individual sequences (w), anticipatory activation (b), past residual activation $(c)$, activation decay $(d)$, and the time between retrieval of each element $(n)$. Predictions can be made about whether the model is more anticipatory or perseveratory in nature based on particular increases or decreases of each parameter.

An important prediction emerging from Dell et al.’s (1997) model is that any factors that result in a largely error-prone system will elicit errors that are perseveratory 
in nature, whereas error-free systems will be mainly anticipatory in nature. This is because events that increase the activation of the present (and therefore lower the error rate) also increase the activation of the future; therefore the relative activation of the future increases at the expense of the relative activation of the past and the anticipatory tendency of the system increases. In contrast, events that increase the activation of the past (and therefore raise the error rate) do not increase the activation of either the present or future; therefore the relative activation of the past increases at the expense of the present and future and the perseveratory tendency of the system increases. The AP, therefore, should be predictable from the overall error rate, regardless of what factors are at play. This is the general anticipatory effect.

The OSCAR model of speech production (Vousden et al., 2000) is concerned with the sequencing and syllabic structuring of phonemes in an utterance. In brief, an intrinsically dynamic control signal (Brown, Preece, \& Hulme, 2000; cf. Houghton, 1990; Houghton \& Hartley, 1996), called a phonological-context signal, determines the activation of a sequence of phonemes such that they are simultaneously partially activated as a function of their relative temporal position. The phonological-context signal consists of a set of oscillators that change over time under their own dynamics and has the property that temporally close states of the signal are more similar than temporally distant states. This signal specifies the metrical structure of the utterance to be spoken, and the constituent phonemes become associated with successive states of the phonological-context signal prior to output. Each phoneme becomes associated with a different state, but one that is similar to nearby states. Phonemes therefore become simultaneously activated as a function of their relative temporal positions; phonemes that 
are nearby are more strongly co-activated than phonemes that are far apart. For production, the phonological-context signal is reinstated and successive states are again generated under the context signal's own dynamics. As each state of the phonologicalcontext signal is regenerated, the phoneme with which that state was initially associated is activated, and nearby phonemes are also activated to the extent that their associated states of the phonological-context signal are similar. A "switch-off” mechanism (postoutput suppression) suppresses the current phoneme after output so that the next phoneme in the sequence can be selected. The amount of suppression an output phoneme receives wears off over subsequent outputs so that it can be output again in the future. Thus upcoming phonemes are activated due to the similarity of nearby states of the phonological-context signal, and previously output phonemes are deactivated by postoutput suppression. Parameters govern the rate and amount of post-output suppression in the model, the amount of time elapsed between successive states of the phonologicalcontext signal (speech rate), and the amount of output noise during production. The output noise parameter determines the proportion of output attempts that are noisy, and therefore prone to error. Noisy output attempts mean that associations between the phonological-context signal and phonemes interfere with each other, with the probability of any two associations interfering being proportional to the similarity of the states of the phonological-context signal. Therefore under noisy output conditions the phonologicalcontext signal is a less strong cue for the correct phoneme, and phonemes that were associated with similar states of the phonological-context vector can be output in error.

An increase in output noise results in an increased error rate and a decrease in AP. This is because when the probability of error in the system is low, the correct phonemes 
are mostly very strongly cued and have sufficient activation to overcome any residual suppression (if they have already been output). If a phoneme has been anticipated, it is likely to be output again (thus completing the anticipation) because in its correct position it is strongly cued by the phonological-context signal, receiving enough activation to overcome suppression. A correctly output phoneme is less likely to be perseverated because in its incorrect (perseverating) position it receives no further activation to overcome its suppression and it must compete with the strongly cued correct phoneme for that position that has not yet been suppressed. Thus when the error rate is low it is relatively harder to perseverate than anticipate, and the system is anticipatory in nature. However, when the probability of error in the system is high, the correct phonemes are often only weakly cued, effectively reducing the amount of post-output suppression that previously output phonemes must overcome to be output again. Therefore, relative to a non noisy output attempt, a correctly output phoneme is a relatively stronger competitor in a perseverating position because the correct phoneme for that position is more weakly cued. This pattern of errors emerges as a property of the model's mechanism itself and therefore provides a detailed explanation of the general anticipatory effect at a mechanistic level.

\section{Age-Related Variation in Performance}

One factor affecting the error rate, which is of interest here, is age-related variation in performance. If the general anticipatory effect applies to age-related variation in performance, then to the extent that young children and older adults make more errors than young adults, proportionally more of their errors should be perseveratory in nature. Evidence shows that young children make many speech errors 
(Jaeger, 1992; Stemberger, 1989), yet few studies have compared the error rates from children’s speech with that of adults (Warren, 1986; Wijnen, 1992). Where this comparison has been made, the findings have been mixed, with some evidence that children err more than adults (Wijnen, 1992), and some evidence to the contrary (Warren, 1986). To summarize, although there is evidence that shows that young children (Wijnen, 1992) and older adults (James, 2000; MacKay \& James, in press) have higher error rates in speech production than young adults, there are few systematic data concerning the link between error rates and AP across different age groups. In the current study, we examined whether the AP can be predicted from age-related variation in performance. To make the comparison of error rates and types transparent, we used the same experimental paradigm to elicit speech errors from young children aged 8 to 11, young adults, and older adults. The tongue twister methodology used previously by Dell et al. (1997) and Schwartz et al. (1994) provides an ideal paradigm for our current purpose. It is effective in eliciting many speech errors, specifically anticipations and perseverations, without placing a great load on memory or perceptual processes.

\section{Experiment 1}

The aim of the first experiment was to test the general anticipatory effect with respect to speech errors elicited from young children and adults. We also included the speech rate manipulation used by Dell et al. (1997), Experiment 3. This provided an additional test of the general anticipatory effect for children's speech and also served as a replication of the experiment by Dell et al. for the adults. The current experiment was similar to Dell et al.’s Experiment 3, but adapted to accommodate children. Some studies concerning speech errors of children have found children to be more error prone than 
adults (e.g., Wijnen, 1992). We expected that children would make more errors overall than adults, and if the general anticipatory effect holds, then a higher proportion of them would be perseveratory.

\section{Method}

Participants. Three different age groups of participants completed the experiment: 20 8- and 9-year-olds (mean age 8 years 7 months) formed the youngest group, 2011 year-olds (mean age 11 years 9 months) formed the older group of children, and 20 undergraduates from the University of Warwick took part as the adult group.

Materials. Listed in Appendix A are twelve of the phrases described in Experiments 1-3 of Dell et al. (1997), along with a new practice example (of a similar format to the 12 main phrases). The main phrases were constructed to make them tongue twisters by using two similar word onsets in four content words to make a meaningful phrase that is difficult to say (e.g., "chef's sooty shoe soles”). These tongue twisters provided the materials for the current experiment. In order to make the task more manageable for children, four of the tongue twisters originally used by Dell et al. were dropped from the current study ("Floyd's fourth floor fort”, "Pam's plain plaid pan", "Freida's fabulous freaky fabrics", and "brief beastly beach breezes"), having been previously judged in a pilot study as being the most problematic for children to say. The twelve tongue twisters were split into two groups of six (Group A and Group B) so that each group was equally error prone (based on a previous pilot study). Each of the phrases was presented in size 46 Times New Roman font on white A4 card in landscape orientation. 
A metronome was used to control the pace at which the tongue twisters were produced.

Design and Procedure. Participants were tested individually in a quiet room. The experiment began with the presentation of the practice phrase. A metronome was set to the very slow rate of 0.8 beats/second and the experimenter repeated the practice phrase at this rate, ensuring a spoken rate of one word per beat. Participants repeated the phrase at the same speed. The metronome was then set to a normal speaking rate of 1.73 beats/second and the practice phrase was held up for 5 seconds for participants to memorize. The phrase was removed and participants repeated the phrase twice, in time with the metronome, clapping twice in between each repetition. The importance of keeping up with the metronome was emphasised to participants, even if in trying to do so they made errors. This procedure was repeated for one of the groups of tongue twisters, completing one trial block at the slow rate. Thus each trial block consisted of repeating each tongue twister in one of the groups twice, at a particular speech rate. Two further trial blocks were completed in a similar manner, except that the practice phrase was omitted, and participants were not required to repeat each phrase at the very slow rate of 0.8 beats/second. For each trial block, the tongue twisters were presented in random order.

The same procedure (including presentation of the practice phrase) was then repeated over 3 trial blocks for the remaining group of tongue twisters, with the metronome set to the fast rate of 2.83 beats/second. Half of the participants in each age group first produced Group A tongue twisters at the slow rate and then Group B tongue twisters at the fast rate. The other half of the participants in each age group first 
produced Group B tongue twisters at the slow rate and then Group A tongue twisters at the fast rate.

Error Coding. Tongue twisters containing at least one error were identified and transcribed. Two experimenters independently categorised the errors according to Dell et al.'s (1997) criteria, replicated here in Appendix B. Any differences in coding were resolved by discussion prior to analysis. The error categories were sound anticipation, sound perseveration, sound exchange, sound anticipation-perseveration, word anticipation, word perseveration, word exchange, word substitution, and other. Examples of errors in each category are shown in Table 1. According to the categorisation rules, errors can involve single phonemes or contiguous groups of phonemes smaller than a word stem. Thus errors such as "pastic plotted...” for "plastic potted..." can be classified as exchanges rather than as perseverative shift errors where the 1 in plastic shifts to potted (see Appendix B, rule 3, for more details).

\section{INSERT TABLE 1 ABOUT HERE}

Results

The data were collapsed across trial blocks to obtain the total number of errors for each participant. The 8-year-olds made most errors, erring at a rate of 18.9 errors per 100 words. The 11-year-olds erred at a rate of 8.01 errors per 100 words, leaving adults as the most error free group erring at a rate of 4.44 errors per 100 words. Table 2 shows the mean number of errors made by each age group overall, and also separately at the slow and fast speech rates.

INSERT TABLE 2 ABOUT HERE 
An analysis of variance (ANOVA) on the mean number of errors was conducted with age (8-years-old, 11-years-old, adult) as the between-subjects variable and speech rate (slow, fast) as the within-subjects variable. The main effect of age was significant, $F(2,57)=76.96, p<.001$. Planned contrasts showed that the 8-year-olds made more errors than the 11-year-olds, $t(19)=8.21, p<.001$, who made more errors than the adults, $t(19)=5.05, p<.001$. The main effect of rate was also significant, $F(1,57)=$ $10.11, p<.01$, showing that overall more errors were made at the fast speech rate than the slow rate. Post-hoc $t$-tests for each age group comparing fast and slow speech rates (corrected for multiple tests of comparison using Bonferroni’s correction) revealed that only 11-year-olds made significantly more errors at the fast speech rate, $t(19)=-3.01, p<$ .01 ; the difference in number of errors made at slow and fast speech rates was not significant for either 8-year-olds or adults. The interaction between age and speech rate was not significant, $F<1$.

To calculate the AP, the numbers of anticipations and perseverations for each participant were counted, collapsing across word and sound errors. In calculating the AP of errors for each participant at each speech rate, it was found that some participants (mainly in the adult group at the slow rate) made no anticipation or perseveration errors, making the calculation of the AP problematic. This resulted in 2 missing data points for the 11-year-olds and 6 missing data points for the adults. However, by collapsing across speech rate, calculation of the AP was possible for all data points. From these totals, calculation of the AP for the three age groups revealed that 8-year-olds had the lowest AP 
of errors, followed by 11-year-olds, and adults had the highest AP (see overall means in Table 2).

A one-way ANOVA on the AP with age (8-years-old, 11-years-old, adult) as the between-subjects variable was conducted. The result of the ANOVA showed a main effect of age, $F(2,57)=4.24, p<.05$. Planned contrasts showed that adults had a significantly higher AP than 8-year-olds, $t(19)=-2.59, p<.01$, and 11-year-olds, $t(19)=$ -2.98, $p<.01$, but that the AP of 8-year-olds was not significantly different from that of the 11-year-olds.

$T$-tests for the child age groups comparing fast and slow speech rates (corrected for multiple tests of comparison using Bonferroni's correction) revealed that only 11year-olds had a significantly lower AP at the fast speech rate, one-tailed $t(17)=2.92, p<$ .01; the difference in the AP made by 8-year-olds was not significant. Because many of the adults produced few or no anticipations and perseverations, calculation of the AP at the slow and fast speech rate was based on the group totals of anticipations and perseverations. Calculating the AP in this way has the advantage that it reduces the effects of large fluctuations in individual APs that arise when there are few data from which to make a calculation. Although calculating the AP in this way is a more appropriate measure when there are few data from individual participants, it is not possible to make a formal comparison between the slow and fast speech rate conditions. Numerically, however, adults had a higher AP in the slow speech rate condition than in the fast speech rate condition (see Table 2), which is the right pattern of results to support the anticipatory speech rate effect. Thus even though the data do not provide formal 
evidence for the anticipatory speech rate effect, they indicate that it is not contradicted either.

\section{Discussion}

The first important finding from Experiment 1 is that when experimentally induced to make speech errors, children made more errors than adults. This is consistent with studies of spontaneous speech errors from children (e.g., Wijnen, 1992). A closer inspection of the data showed a developmental trend in error rates with the youngest participants making the most errors while the oldest made the least.

Second, this experiment also revealed that overall the AP made by adults was higher than that of children. The anticipatory age effect states that as age increases from childhood through to adulthood, the anticipatory proportion of speech errors should increase accordingly. The AP of errors from 8-year-olds and 11-year-olds did not differ significantly, although the adult group had a significantly higher AP than both of the children's groups. However, while the difference in the AP from 8- and 11-year-olds failed to reach significance, it is clearly in the predicted direction. Therefore the results provide evidence for an anticipatory age effect.

Third, there was at least partial support for the anticipatory speech rate effect, which states that the AP will be greater at slower speech rates. Thus, 11-year-olds showed the expected anticipatory speech rate effect, with a significantly higher error rate and a significantly lower AP at the fast compared with the slow speech rate. However, the difference in AP at the fast and slow rates for the 8-year-olds failed to reach significance. Why do 11-year-olds' errors reflect the anticipatory speech rate effect but not the 8-year-olds'? If the general anticipatory effect holds true then the AP should only 
decrease if the error rate increases. The results showed that while this was the case for 11-year-olds, the 8-year-olds did not produce significantly more errors at the fast rate (perhaps because their errors at the slow rate were already quite high), so there should be no significant decrease in the AP either. Although the anticipatory speech rate effect was not significant for the 8-year-olds, their pattern of errors at each rate fit the appropriate pattern predicted by the anticipatory speech rate effect, i.e., decreased AP at faster speech rates.

In the case of the adults, it was not possible to test for an anticipatory speech rate effect because they made few anticipations and perseverations overall, especially at the slow speech rate. The mean number of anticipation and perseveration errors per participant at the slow rate was just 3.6, with 4 participants making no anticipation or perseveration errors at all. Calculation of the AP based on such few errors is bound to be very noisy because the AP as a measure is not sensitive enough to capture any bias for small numbers of errors. For this reason, the AP values for adults were calculated from the group totals of anticipations and perseverations, which rendered a formal comparison of the AP at slow and fast speech rates impossible for adults. However, numerically, the AP decreased with speech rate and the error rate increased with speech rate, which is the same pattern predicted by the anticipatory speech rate effect. The adult data are therefore generally supportive of an anticipatory speech rate effect even though it was not formally evaluated.

In Experiment 1, the materials and procedure were adjusted to accommodate the reduced concentration span of children. Hence it would have been difficult to increase the number of trials for adult participants alone without running into issues of differential 
practice effects across age groups. In Experiment 2, we tested adults only and we increased the number of tongue twisters and trials that participants completed in order to increase the number of speech errors.

\section{Experiment 2}

The aim of Experiment 2 was to test the general anticipatory effect with respect to speech errors elicited from older adults in addition to young adults. The few studies concerning speech errors of older adults have found them to be more error prone than young adults (James, 2000; MacKay \& James, in press). We therefore expected in this experiment that older adults would make more errors overall than young adults, and if the general anticipatory effect holds, then a higher proportion of them would be perseveratory. We also included the speech rate manipulation from Experiment 1 . As there were insufficient data to satisfactorily test the anticipatory speech rate effect with adults in Experiment 1, we increased the numbers of both tongue twisters and trials in Experiment 2.

Method

Participants. There were 22 older participants aged between 65 and 84 years (10 female and 12 male, mean age 72 years, 7 months), and 20 undergraduates from the University of Warwick in the young adults group (9 female and 11 male, mean age 21 years). The older participants were recruited from a participant pool of older people developed at the University of Warwick and had taken part in psychology experiments previously. They were required to make their own travel arrangements to attend a testing session at the University of Warwick, situated at least two miles from the nearest town or city. 
Table 3 contains a summary of the background information gathered for all participants (excluding one older participant whose data were deleted from the analyses as described later). Current health, eyesight (with glasses, if worn), and hearing (with a hearing aid, if worn) were assessed by self ratings. Vocabulary was measured with the first part of the Mill Hill Vocabulary test (Raven, Raven, \& Court, 1988), which requires choosing the most appropriate synonym for a target word from a choice of six alternatives. Speech rates for each participant were measured by recording the average time taken over two trials to count aloud from 1 to 20 as quickly as possible.

The self-rated measures for health, eyesight, and hearing were generally high, with average ratings equivalent to good. Older adults gave significantly lower ratings for current health, eyesight, and hearing than the young adults, although there were no selfratings of very poor or poor in either group. There was clearly an age advantage for vocabulary, but an age deficit for speech rate. This pattern of age differences in the background data is consistent with the pattern typically found in the aging literature, that is, a marked decline in speed yet stability or growth in crystallised intelligence, indexed here by vocabulary (see, for example, Salthouse, 1991).

Materials. Listed in Appendix C are all sixteen phrases described in Experiments 1 to 3 of Dell et al. (1997), along with three practice examples (of a similar format as the main phrases). These tongue twisters provided the materials for the current experiment. The sixteen tongue twisters were split into two groups of eight (Group A and Group B) so that each group was equally error prone (based on a previous pilot study). Each of the phrases was presented in size 40 Times New Roman font on white A4 card in landscape orientation. A stopwatch was used to time the counting task. A computer was 
programmed to beep at a rate of 1.73 beats/second for the slow rate, and 2.83

beats/second for the fast rate. The slow rate beeps were then recorded onto one side of an audio tape, and the fast rate beeps were recorded onto the other side.

Design and Procedure. Participants were tested individually in a quiet room. At the start of the session participants were timed while they counted aloud from 1 to 20 as quickly as possible. This was repeated once more and the average of the two trials was recorded.

The experiment began with a short practice session. Practice began with the presentation of one of the practice phrases. The practice phrase was held up for 5 seconds for the participant to memorize. The audio tape played the slow beep at the rate of 1.73 beats/second. The phrase was removed and the participant repeated the phrase twice, in time with the beep from the audio tape, leaving two beats in between each repetition. This procedure was repeated for the remaining two practice phrases. The importance of keeping up with the beep from the audio tape was emphasised to participants, even if in trying to do so they made errors.

The experiment proper then began with the presentation of tongue twisters from one of the groups, completing one trial block at the slow rate. Each trial block consisted of repeating each tongue twister in one of the groups twice, at a particular speech rate. Three further trial blocks were then completed in the same manner. For each trial block the tongue twisters were presented in random order. The same procedure was repeated over four trial blocks for the other group of tongue twisters, with the audio tape playing the fast beep at a rate of 2.83 beats/second. Half of the participants in each age group first produced Group A tongue twisters at the slow rate and then Group B tongue twisters 
at the fast rate. The other half of the participants in each age group first produced Group B tongue twisters at the slow rate and then Group A tongue twisters at the fast rate. Error Coding. Error transcription and coding was carried out according to the same method and rules described in Experiment 1, except that all coding was carried out by one experimenter.

Results

Many of the older participants failed to keep up with the fast speech rate, set at 2.83 beats/second. The criterion for keeping up with the rate was that each tongue twister had to be repeated in four consecutive beats of the audio tape. None of the elderly participants was able to meet the criterion for all tongue twisters, and many failed to keep up on over $80 \%$ of all the tongue twisters in the fast condition. Given that the older adults spoke at a significantly slower rate than the young adults (see Table 3), it is likely that the increase in speech rate from slow to fast was too great for them. Therefore only the errors from the slow speech rate condition of older adults were analysed. One of the older participants failed to keep up with both the fast and the slow speech rates and her data were not included in any of the analyses, leaving data from 41 participants (20 young; 21 older).

The data were collapsed across trial block to obtain the total number of errors for each participant. At the slow rate, older adults made more errors, erring at a rate of 4.7 errors per 100 words, in comparison with the young adults who erred at a rate of 3.9 errors per 100 words. At the fast rate, young adults made 5.7 errors per 100 words. Table 4 shows the mean number of errors made by the young adults at the slow and fast rates and the mean number of errors made by the older adults at the slow rate. 


\section{INSERT TABLE 4 ABOUT HERE}

Young and older adults did not make significantly different numbers of errors at the slow speech rate, $t(39)=0.92$. A paired $t$-test comparing the mean number of errors made by the young adults at the slow and fast speech rates was significant, $t(19)=2.67, p$ $<.05$, showing that the young adults made more errors at the fast speech rate than at the slow speech rate.

The number of anticipations and perseverations for each participant was counted, collapsing across word and sound errors. At the slow rate, four young adults made no errors, preventing a calculation of the AP. The following analyses are therefore based on the data of 16 young adults. Table 4 shows the AP of the young adults at both the slow and fast rates, and the AP of the older adults at the slow rate. Young and older adults did not differ significantly in their APs at the slow speech rate, $t(35)=0.31, p>.05$. A paired $t$-test comparing the AP of the young adults at the slow and fast speech rates was significant, one-tailed $t(15)=2.06, p<.05$, showing that young adults had a significantly lower AP at the fast speech rate than at the slow speech rate. ${ }^{\mathrm{i}}$

\section{Discussion}

Experiment 2 demonstrated that in the slow condition, older adults did not make significantly more errors than young adults. This result is not consistent with the small number of studies where older adults’ speech errors have been reported (James, 2000; MacKay \& James, in press). It is surprising that the older adults did not make more errors than the young adults because the older adults were clearly slower than the young 
adults in their maximum speech rate, consistent with the aging literature. Therefore, in comparison to the young adults, the slow speech rate condition for the older adults should have been relatively more demanding. It is therefore puzzling that the older adults did not make more errors than the young adults in Experiment 2. However, many aspects of language use are preserved in aging (Burke \& MacKay, 1997; Burke, MacKay, \& James, 2000). This is also seen here in an age advantage for vocabulary. But it must be noted that where there are age-related deficits for language, they are generally in production rather than in comprehension (Burke \& MacKay, 1997; Burke et al., 2000). Older adults in this experiment did, of course, show an age deficit in production in their inability to keep pace with the beat at the fast rate. Many of the older adults omitted to produce each word on the beat, and instead, for example, left one beat in between each word. These hesitations and pauses may have been attempts to prevent a different type of error from occurring, which is why it was not possible to analyse the data from the older adults in the fast speech rate condition. When older adults do largely keep up with the fast beat and unwanted hesitations and pauses are coded as omission errors, data from an unpublished study show an age increase in omission errors, but not other error types (D. M. Burke, personal communication, 2 July 1999). This is consistent with other studies that show an age-related deficit in omission errors during production tasks (James, 2000). The anticipatory speech rate effect states that the AP will be greater at slower speech rates. The results from this experiment clearly show this to be the case for young adults; thus, errors were significantly fewer and the AP significantly higher at the slow than at the fast speech rate. This is consistent with previous findings (Dell et al., 1997), and also with the children's data from Experiment 1. It was not possible to test the 
anticipatory speech rate effect for older adults because they were unable to keep pace with the beat at the fast speech rate and so their data in that condition were not analysed.

The findings of this experiment also revealed that at the slow speech rate the AP was not significantly different for the young and older adults. An anticipatory aging effect would appear as a decrease in the anticipatory proportion of speech errors as young adults age in line with an increase in errors due to aging. In fact, there was no significant increase in errors with aging, and so the lack of a difference in AP for young and older adults is consistent with the lack of an age effect on errors. Therefore the results from this experiment are not inconsistent with an anticipatory age effect because a null age effect on errors predicts a null age effect on AP. If there had been an effect of age on AP in the context of a null effect of age on errors, then this would have been evidence against an anticipatory age effect.

To our knowledge, only one other study has examined the anticipation and perseveration speech errors of older adults (MacKay \& James, in press). However, direct comparison with MacKay and James’s study is problematic for methodological and classification reasons. We return to this issue in the general discussion.

\section{General Discussion}

In Experiment 1, speech errors were elicited from 8- and 11-year-old children, and young adults when they produced tongue twisters at slow and fast speech rates. Error rates decreased and the AP increased from children to young adults, supporting an anticipatory age effect from children and young adults. In Experiment 2, the same methodology was used to elicit speech errors from young and older adults, again at slow 
and fast speech rates. Neither error rate nor AP differed significantly between young and older adults. In cases where fast speech resulted in a significantly higher error rate than slow speech, the AP was significantly lower. To summarise, we have replicated the anticipatory speech rate effect with children and young adults, and an age manipulation has provided support for Dell et al.'s (1997) predictions from speech error data across the lifespan.

The General Anticipatory Effect

According to Dell et al. (1997), the AP can be predicted from the overall error rate, regardless of the variable causing variation in performance. This is the general anticipatory effect. In the two experiments presented here, age and speech rate manipulations have resulted in a variety of performance levels. To test the general anticipatory effect with an age manipulation that spans children to older adults, Figure 1 shows the AP plotted as a function of the log of the error probability (cf. Dell et al.) for each age group for the fast and slow speech rates, where available.

\section{INSERT FIGURE 1 ABOUT HERE}

The best fitting linear function shows that the AP decreases significantly with the overall error rate (slope $=-.254$, intercept $=.206), r=-.617, p<.05$ (one-tailed), supporting a general anticipatory effect across Experiments 1 and 2. The regression line in Figure 1 is similar to the regression line fitted from the data from Experiment 3 in Dell et al. (1997) (slope $=-.193$, intercept $=.185)$, which was based on variation over speech rate and practice in young adults. Thus to obtain such a similar regression line from data 
based on variation over speech rate and age further supports a general anticipatory effect. It is interesting to note that the anticipatory speech rate effect (seen in Figure 1 as dashed lines connecting data points for the slow and fast speech rate conditions within each age group) appears to be more pronounced than the anticipatory age effect. Also of interest is the fact that the anticipatory speech rate effect data for young adults from both experiments lie almost on the same line, with both fast conditions to the right of, and below, the slow conditions, in line with the general anticipatory effect.

\section{Anticipatory Effects in Music Performance}

We note that evidence for the general anticipatory effect is also found in production tasks from other domains, such as piano playing. Drake and Palmer (2000) examined anticipation and perseveration errors from pianists who performed short pieces of unfamiliar Western tonal music. They found that error rates and AP varied according to skill level and practice, consistent with the general anticipatory effect, such that more advanced performers and more practiced trials tended to have a higher AP and fewer errors. In a later study, Palmer and Pfordresher (2003) used the same experimental design as Dell et al.’s (1997, Experiment 3) but adult participants performed musical pieces at a medium and fast tempo instead of repeating tongue twisters. Again, evidence for the general anticipatory effect was found with respect to practice in that trials that represented more practice tended to have fewer errors and a higher AP. It therefore appears that the general anticipatory effect is not necessarily specific to the language domain, but that it can be found in other domains where production of serially-ordered information is fundamental. 
However, while evidence of a general anticipatory effect has been found in piano playing from manipulations of skill level and practice, there was no evidence of an anticipatory rate effect when adult pianists played pieces at a medium and fast tempo (Palmer \& Pfordresher, 2003). In a second experiment with children, Palmer and Pfordresher again failed to find evidence of an anticipatory rate effect. They suggest that pianists adapt to faster production rates in piano playing by anticipating more, and that anticipatory priming is not invariant across production rate, as suggested by Dell et al. (1997). The demonstration of the anticipatory rate effect in the experiments reported here is important because it reinforces support for an anticipatory production rate effect, at least in speech. The discrepancy between how variation in production rate is handled in speech and music performance raises an interesting question of why pianists are able to anticipate more at faster production rates, yet speakers apparently are not.

The findings from the experiments reported here are compatible with both Dell et al.’s (1997) and Vousden et al.’s (2000) models. Both models have previously demonstrated their ability to explain the anticipatory speech rate effect. The present study was not designed to distinguish between the two models; however, it is useful to consider how each model accounts for the anticipatory age effect.

\section{Children's Errors}

The finding of an anticipatory age effect for children and young adults is accounted for in Dell et al.'s (1997) model by proposing that children have less knowledge of phoneme sequences than adults. In their model, sequential knowledge is represented by parameter $w$. Dell et al. have shown that as $w$ increases, the AP increases as well. As Dell et al. note, alternative accounts for the anticipatory age effect are 
possible in their model. For example, there is evidence to suggest that the ability to inhibit past activation develops across childhood (Dempster, 1992; Diamond, 2002; Diamond, Kirkham, \& Amso, 2002; Gerstadt, Hong, \& Diamond, 1994). In Dell et al.'s model, this can be simulated by an increase in parameter $c$, which results in more errors and a lower AP. Such an explanation would be consistent with Stemberger’s (1989) interpretation of children's speech errors in which a greater number of perseveration errors is accounted for by a slower decay of activation. A reduction in the amount of post-output suppression in Vousden et al.'s (2000) model would also predict an increase in errors and a decrease in the AP.

Although the data presented here cannot distinguish between alternative accounts in Dell et al.'s model, we note that when McCormack, Brown, Vousden, and Henson (2000) measured levels of response inhibition in children in a short-term memory for serial order task, they found that response inhibition remained developmentally invariant. Likewise for older adults, Maylor and Henson (2000) found no evidence of reduced response inhibition in a similar short-term memory for serial order task. Hence there is evidence to the contrary that response inhibition changes according to age, at least in tasks requiring short-term memory for serial order. It could be argued that the sequence processing aspects of speech production are more similar to the short-term memory for serial order tasks than other studies (Dempster, 1992; Diamond, 2002; Diamond, Kirkham, \& Amso, 2002; Gerstadt, Hong, \& Diamond, 1994) that have found evidence for reduced response inhibition in childhood, relative to adulthood. To the extent that speech production and short-term memory for serial order tasks tap into similar sequencing processes, we find it unlikely that an anticipatory age effect, at either end of 
the lifespan, is best explained by a reduction in response suppression. Recently, Gershkoff-Stowe (2002) has also argued against a reduced response suppression account of developmental changes in speech errors. Results from Gershkoff-Stowe's study show that perserveratory naming errors were common in children aged 16 and 28 months of age when both groups of children were presented with unfamiliar objects to name. The results are instead interpreted as supporting an account whereby the relative activation strength of individual words increases with age. Thus Gershkoff-Stowe's account is consistent with Dell et al. (1997), who propose that errors decrease due to a developmental increase in sequential knowledge.

Vousden et al.'s (2000) model has not been applied to children’s speech error data; however the most likely sources of effect in the model are located in the quality of the context signal (at encoding or retrieval) or in the post-output suppression process. We note that Brown, Vousden, McCormack and Hulme (1999) have shown that developmental improvements in short-term memory for serial order can be explained using a similar architecture by increasing the temporal distinctiveness of states of the context signal, either at encoding and retrieval, or just at retrieval. Other parameter manipulations (e.g., ones that implemented a reduction in attention at encoding or caused new items to interfere with previously learnt items) could not simulate the developmental pattern of serial order errors in short-term memory. Maylor, Vousden, and Brown (1999) have also shown how age-related deficits in a short-term memory for serial order task can be explained by a deterioration in the distinctiveness of states of the context signal. Considering the findings from short-term memory for serial order studies concerning reduced response inhibition, we therefore expect that an explanation that involves 
manipulating the distinctiveness of states of the context signal will be more fruitful than one that relies on a reduced response inhibition account.

\section{Older Adults’ Errors}

Surprisingly, we did not find any evidence of increased error rates at the slow speech rate in older adults relative to young adults, despite the fact that their maximum speech rate was clearly slower than that of young adults. It is worth noting that the AP was also not significantly different for young and older adults, which is consistent with Dell et al.’s (1997) predictions. Dell et al. point out that the general anticipatory effect should apply to aging whether the age-related deficit is due to slower processing speed (Salthouse, 1985, 1996), information loss (Myerson, Hale, Wagstaff, Poon, \& Smith, 1990), information transmission failure (Burke, MacKay, Worthley, \& Wade, 1991), or reduced inhibitory processing (Hasher, Zacks, \& May, 1999; Zacks \& Hasher, 1994). In our study, there is clear evidence of a speed deficit for older adults for speech production; therefore, the data could have quite plausibly shown a different pattern that would not be consistent with Dell et al. For example, the older adults could have made more errors than the young adults, but the AP may not have been different, or vice versa. Also, previous studies have shown older adults to be more error prone on production tasks (MacKay \& James, 2000). So although the lack of an age difference in error rates was surprising, the lack of a difference in the AP is salient here because it is predictable from the observed error rates.

The present study was not designed to distinguish between the current theories of aging mentioned above, and considering the current findings in terms of these theories does not appear to differentiate between them. It is likely that each theory could 
accommodate the findings, but until the theories are implemented in terms of formal models it is difficult to make precise predictions. In the present study, older participants were unable to keep up with the beat in the fast condition, which could be interpreted as making omission errors in the fast condition. According to a theory of general slowing (e.g., Salthouse, 1996), the speed of cognitive operations should slow at least within a domain to a constant degree, regardless of the task faced. Therefore older participants should exhibit more errors than young adults, perhaps as omission errors if information is available too late in processing. According to a reduced inhibition account (Hasher, Zacks, \& May, 1999; Zacks \& Hasher, 1994), task-irrelevant information cannot be as effectively suppressed in older age. Therefore more errors may result from either semantically or phonologically related words competing with the target word - but this scenario could also result in more omission errors if irrelevant information prevented a candidate word from rising sufficiently in activation above all others. According to an information transmission failure hypothesis (Burke et al., 1991), links in an activation style network are weakened with age so that phonological nodes that rely on one-to-one links would be insufficiently primed to be output. The information transmission failure account would also therefore result in missing information, consistent with an increase in omission errors. Thus the current results do not advance a particular argument for why older participants make more errors than their young counterparts.

It must be noted that a large part of the reported increase in older adults' speech errors in the literature (e.g., James, 2000; MacKay \& James, in press) comes from an increase in omission errors rather than in the contextual substitution errors that we are concerned with here. It appears that speaking in synchrony with a beat set to a fast 
speech rate is very demanding for older adults. However, when older adults do manage to keep pace, there are still many pauses and hesitations, and age differences only appear in the number of omission errors (D. M. Burke, personal communication, 20 June 1999). The present study has therefore highlighted the problems of examining speech errors in older adults using the tongue twister methodology of Dell et al. (1997). Unfortunately, there are no corpora of older adults’ spontaneous speech errors available for comparison.

An alternative method for eliciting speech errors is the transform technique (MacKay \& James, in press), in which participants are required to substitute critical phonemes in a presented word. Using this technique, MacKay and James found an increase in contextual substitution errors for older adults. However, while MacKay and James’s study represents an important investigation of a speech-based serial order task with older participants, its purpose was not to investigate anticipatory effects. There are large differences in methodology, error types and classification, making direct comparison with their study problematic. For example, the transform technique has more potential for perceptual or memory errors than the error induction task used by Dell et al. (1997), where participants simply repeat a tongue twister several times. The classification of speech errors as anticipatory or perseveratory in the transform technique depends on whether the critical phoneme is substituted in the intended location or not, and not on the actual repetition of a phoneme as in Experiments 1 and 2 here. It is also important to note that the only possible types of errors using the transform technique are within-word errors, whereas the reported data on the general anticipatory effect are taken from between-word errors. It is not clear that within-word errors follow the same 
patterns as between-word errors, again making comparisons between the two techniques difficult.

Conclusion

To conclude, we have experimentally elicited speech errors from children, young adults, and older adults using the same paradigm. This allowed us to make formal comparisons of the error rates and AP across the lifespan, providing new supporting evidence for Dell et al’s (1997) general anticipatory effect, and confirmation of an anticipatory age effect through childhood. The findings are consistent with recent models of sequencing in speech production (Dell et al., 1997; Vousden et al., 2000), and provide a starting point for evaluating why such patterns emerge across the lifespan. Future studies will be necessary to differentiate between the causal mechanisms embodied in the models that effect such age-related changes. 


\section{References}

Boomer, D. S., \& Laver, J. D. M. (1968). Slips of the tongue. British Journal of Disorders of Communication, 3, 2-12.

Broeke, Van Den M. P. R., \& Goldstein, L. (1980). Consonant features in speech errors. In V. A. Fromkin (Ed.), Errors in Linguistic Performance: Slips of the Tongue, Ear, Pen, and Hand (pp. 47-65). London: Academic Press.

Brown, G. D. A., Preece, T., \& Hulme, C. (2000). Oscillator-based memory for serial order. Psychological Review, 107, 127-181.

Brown, G. D. A., Vousden, J. I., McCormack, T. M., \& Hulme, C. (1999). The development of memory for serial order: A temporal-contextual distinctiveness model. International Journal of Psychology, 34, 389-402.

Burgess, N., \& Hitch, G. J. (1992). Toward a network model of the articulatory loop. Journal of Memory and Language, 31, 429-460.

Burke, D. M., \& MacKay, D. G. (1997). Memory, language, and aging. Philosophical Transactions of the Royal Society of London, Series B, 352, 1845-1856.

Burke, D. M., MacKay, D. G., \& James, L. E. (2000). Theoretical approaches to language and aging. In T. J. Perfect \& E. A. Maylor (Eds.), Models of cognitive aging (pp. 204-237). Oxford, England: Oxford University Press.

Burke, D. M., MacKay, D. G., Worthley, J. S., \& Wade, E. (1991). On the tip of the tongue: What causes word finding failures in young and older adults? Journal of Memory and Language, 30, 542-579.

Dell, G. S. (1984). Representation of serial order in speech: Evidence from the repeated phoneme effect in speech errors. Journal of Experimental Psychology: Learning, 
Memory, and Cognition, 10, 222-233.

Dell, G. S. (1986). A spreading-activation theory of retrieval in sentence production. Psychological Review, 93, 283-321.

Dell, G. S. (1989). The retrieval of phonological forms in production: tests of predictions from a connectionist model. In W. Marslen-Wilson (Ed.), Lexical Representation and Process (pp. 136-165). Cambridge, MA: MIT Press.

Dell, G. S. (1990). Effects of frequency and vocabulary type on phonological speech errors. Language and Cognitive Processes, 5, 313-349.

Dell, G. S., Burger, L. K., \& Svec, W. R. (1997). Language production and serial order: A functional analysis and a model. Psychological Review, 104, 123-147.

Dell, G. S., \& Reich, P. A. (1981). Stages in sentence production: An analysis of speech error data. Journal of Verbal Learning and Verbal Behavior, 20, 611-629.

Dempster, F. N. (1992). The rise and fall of the inhibitory mechanism - toward a unified theory of cognitive-development and aging. Developmental Review, 12, 45-75.

Diamond, A. (2002). Normal development of prefrontal cortex from birth to young adulthood: Cognitive functions, anatomy, and biochemistry. In D. T. Stuss \& R. T. Knight (Eds.), Principles of frontal lobe function (pp. 466-503). London, UK: Oxford University Press.

Diamond, A., Kirkham, N., \& Amso, D. (2002). Conditions under which young children can hold two rules in mind and inhibit a prepotent response. Developmental Psychology, 38, 352-362.

Drake, C., \& Palmer, C. (2000). Skill acquisition in music performance: relations between planning and temporal control. Cognition, 74, 1-32. 
Fromkin, V. A. (1971). The non-anomalous nature of anomalous utterances. Language, 51, 27-52.

Garcia-Albea, J. E., del Viso, S., \& Igoa, J. M. (1989). Movement errors and levels of processing in sentence production. Journal of Psycholinguistic Research, 18, 145161.

Garnham, A., Shillcock, R. C., Brown, G. D. A., Mill, A. I. D., \& Cutler, A. (1982). Slips of the tongue in the London-Lund corpus of spontaneous conversation. In A. Cutler (Ed.), Slips of the tongue and language production (pp. 251-263). Amsterdam: Mouton.

Garrett, M. F. (1975). The analysis of sentence production. In G. H. Bower (Ed.), The psychology of learning and motivation (Vol. 9, pp. 137-177). New York: Academic Press.

Garrett, M. F. (1976). Syntactic processes in sentence production. In R. J. Wales \& E. Walker (Eds.), New approaches to language mechanisms (pp. 231-255). Amsterdam: North Holland Publishing Company.

Gershkoff-Stowe, L. (2002). Object naming, vocabulary growth, and the development of word retrieval abilities. Journal of Memory and Language, 46, 665-687.

Gerstadt, C. L., Hong, Y. J., \& Diamond, A. (1994). The relationship between cognition and action - performance of children $3 \frac{1}{2} 2^{-7}$ years old on a Stroop-like day-night test. Cognition, 53, 129-153.

Harley, T. A. (1984). A critique of top-down independent levels models of speech production: Evidence from non-plan-internal speech errors. Cognitive Science, 8, 191-219. 
Harley, T. A., \& MacAndrew, S. B. G. (1995). Interactive models of lexicalization: Some constraints from speech error, picture naming, and neuropsychological data. In J. Levy, D. Bairaktaris, J. Bullinaria, \& D. Cairns (Eds.), Connectionist models of memory and language (pp. 311-313). London: UCL Press.

Hasher, H., Zacks, R. T., \& May, C. P. (1999). Inhibitory control, circadian arousal, and age. In D. Gopher \& A. Koriat (Eds.), Attention and performance XVII. Cognitive regulation and performance: Interaction of theory and application (pp. 653-675). Cambridge, MA: MIT Press.

Henson, R. N. A., Norris, D. G., Page, M. P. A., \& Baddeley, A. D. (1996). Unchained memory: Error patterns rule out chaining models of immediate serial recall. The Quarterly Journal of Experimental Psychology, 49A, 80-115.

Houghton, G. (1990). The problem of serial order: A neural network model of sequence learning and recall. In R. Dale, C. Mellish, \& M. Zock (Eds.), Current research in natural language generation (pp. 287-319). London: Academic Press.

Houghton, G., \& Hartley, T. (1996). Parallel models of serial behaviour: Lashley revisited. Psyche, 2, available: http://psyche.cs.monash.edu.au/V2/psyche-2-25houghton.html.

Jaeger, J. J. (1992). “Not by the chair of my hinny hin hin”: Some general properties of slips of the tongue in young children. Journal of Child Language, 19, 335-366.

James, L. E. (2000). Speech errors of young and older adults fit Node Structure Theory. Paper presented at the Cognitive Aging Conference, Atlanta, GA.

Lashley, K. S. (1951). The problem of serial order in behaviour. In L. A. Jefress (Ed.), Cerebral mechanisms in behaviour: The Hixon Symposium (pp. 112-146). New 
York: Wiley.

MacKay, D. G. (1970). Spoonerisms: The structure of errors in the serial order of speech. Neuropsychologia, 8, 323-350.

MacKay, D. G. (1978). Speech errors inside the syllable. In A. Bell \& J. B. Hooper (Eds.), Syllables and segments. Amsterdam: North Holland Publishing.

MacKay, D. G. (1987). The organisation of perception and action. New York: Springer Verlag.

MacKay, D. G., \& James, L. E. (in press). Sequencing, speech production, and selective effects of aging on phonological and morphological speech errors. Psychology and Aging.

Maylor, E. A., \& Henson, R. N. A. (2000). Aging and the Ranschburg effect: No evidence of reduced response suppression in old age. Psychology and Aging, 15, 657-670.

Maylor, E. A., Vousden, J. I., \& Brown, G. D. A. (1999). Adult age differences in shortterm memory for serial order: Data and a model. Psychology and Aging, 14, 572594.

McCormack, T. M., Brown, G. D. A., Vousden, J. I., \& Henson, R. N. A. (2000). Children's serial recall errors: Implications for short-term memory development. Journal of Child Experimental Psychology, 76, 222-252.

Myerson, J., Hale, S., Wagstaff, D., Poon, L. W., \& Smith, G. A. (1990). The information loss model: A mathematical theory of age-related cognitive slowing. Psychological Review, 97, 475-487.

Nooteboom, S. G. (1969). The tongue slips into patterns. In A. G. Sciarone, A. J. v. 
Essen, \& A. A. v. Raad (Eds.), Nomen: Leyden studies in linguistics and phonetics (pp. 114-132). The Hague: Mouton.

Nooteboom, S. G. (1980). Speaking and unspeaking: Detection and correction of phonological and lexical errors in spontaneous speech. In V. A. Fromkin (Ed.), Errors in linguistic performance: Slips of the tongue, ear, pen, and hand (pp. 8795). London: Academic Press.

Palmer, C., \& Pfordresher, P. Q. (2003). Incremental planning in sequence production. Psychological Review, 110, 683-712.

Raven, J. C., Raven, J., \& Court, J. H. (1988). The Mill Hill vocabulary scale. London: H. K. Lewis.

Rumelhart, D. E., \& Norman, D. A. (1982). Simulating a skilled typist: A study of skilled cognitive motor performance. Cognitive Science, 6, 1-36.

Salthouse, T. A. (1985). A theory of cognitive aging. Amsterdam: North Holland Publishing.

Salthouse, T. A. (1991). Theoretical perspectives on cognitive aging. Hillsdale, NJ: Erlbaum.

Salthouse, T. A. (1996). The processing-speed theory of adult age differences in cognition. Psychological Review, 103, 403-428.

Schwartz, M. F., Saffran, E. M., Bloch, D. E., \& Dell, G. S. (1994). Disordered speech production in aphasic and normal speakers. Brain and Language, 47, 52-88.

Shattuck-Hufnagel, S. (1979). Speech errors as evidence for a serial-ordering mechanism in sentence production. In W. E. Cooper \& E. C. T. Walker (Eds.), Sentence processing: psycholinguistic studies presented to Merrill Garrett (pp. 295-342). 
London: Erlbaum.

Shattuck-Hufnagel, S. (1987). The role of word-onset consonants in speech production planning: New evidence from speech error patterns. In E. Keller \& M. Gopnik (Eds.), Motor and sensory processes of language (pp. 17-51). Hillsdale, NJ: Erlbaum.

Shattuck-Hufnagel, S., \& Klatt, D. H. (1979). The limited use of distinctive features and markedness in speech production: Evidence from speech error data. Journal of Verbal Learning and Verbal Behavior, 18, 41-55.

Stemberger, J. P. (1982). The nature of segments in the lexicon: Evidence from speech errors. Lingua, 56, 235-259.

Stemberger, J. P. (1985). An interactive activation model of language production. In A. W. Ellis (Ed.), Progress in the psychology of language (Vol. 1, pp. 143-186). London: Erlbaum.

Stemberger, J. P. (1989). Speech errors in early child language production. Journal of Memory and Language, 28, 164-188.

Stemberger, J. P. (1990). Wordshape errors in language production. Cognition, 35, 123157.

Vousden, J. I., Brown, G. D. A., \& Harley, T. A. (2000). Serial control of phonology in speech production: A hierarchical model. Cognitive Psychology, 41, 101-175.

Warren, H. (1986). Slips of the tongue in very young children. Journal of Psycholinguistic Research, 15, 309-344.

Wickelgren, W. A. (1969). Context-sensitive coding, associative memory, and serial order in (speech) behaviour. Psychological Review, 76, 1-15. 
Wijnen, F. (1992). Incidental word and sound errors in young speakers. Journal of Memory and Language, 31, 734-755.

Zacks, R. T., \& Hasher, H. (1994). Directed ignoring: Inhibitory regulation of working memory. In D. Dagenbach \& T. H. Carr (Eds.), Inhibitory processes in attention, memory, and language (pp. 241-264). San Diego, CA: Academic Press. 


\title{
Appendix A
}

\section{Tongue twisters used in Experiment 1}

\section{Practice Example}

Lucy Locket’s little lamb

\author{
Group A Tongue Twisters \\ chef's sooty shoe soles \\ thirty three throbbing thumbs \\ Gloria’s Greek green gloves \\ Bonnie’s brown bread box \\ fine fresh free fish \\ simple slender silver slippers
}

\section{Group B Tongue Twisters}

Danny’s dripping dish drain

plastic potted pansy plants

Brad's burned bran buns

Tike’s tricky trike tire

five frantic fat frogs

sappy shiny shop signs 


\section{Appendix B}

Error categorisation rules for Experiments 1 and 2 (reproduced from Appendix B, pp

145-146 of Dell et al., 1997)

Categories

Word anticipation (WA), word perseveration (WP), word exchange (WE), word substitution (WS), sound anticipation (SA), sound perseveration (SP), sound exchange (SE), sound anticipation-perseveration (SAP).

1. Word versus sound. An error is a word error if the error string is a word or word stem from elsewhere in the utterance (contextual word error), or if it is a word from outside the utterance that could not have arisen from the movement of sounds within the utterance (WS category). Otherwise, the error is a sound error, provided that it doesn't fall in the $\mathrm{O}$ category as specified in (2).

Examples: “Tike’s tricky trike tire” spoken as “trike’s tricky trike tire” is a word error because trike is a word from the phrase, even though the change is small. "Sappy shiny shop signs” spoken as "sappy shiny stop signs” is a WS. "Chef's sooty shoe soles” spoken as “chef's sooty sue soles” is a sound error, even though sue is a word. If the sound /s/ replaces the /sh/ in shoe, the result is sue.

2. The "other" category. Errors involving grammatical affixes ("chef's sooty shoes sole” for “chef's sooty shoe soles”), deletion of a word (“chef's sooty shoe” ), or the noncontextual substitution of a sound ("chef's sooty foo soles” ) were placed in the O category. 
3. Serial-order errors. All sound and word errors outside of the WS and O categories are counted as serial-order errors, either as anticipations, perseverations, exchanges, or anticipation-perseverations. For word errors, the error was categorised as WA, WP, or WE depending on the location of the intruding word (for WA vs. WP) and whether there was a corresponding substitution in the same utterance (WE).

Examples: "Brad's burned brad buns” is WP. "Chef's sooty sole soles” is WA. "Brad's bran burned buns" is WE.

For sound errors, the target and intruding sounds are first defined. These can be single phonemes or contiguous groups of phonemes smaller than a word stem. Errors in which singleton consonants interacted with clusters (e.g., "plastic potted" is spoken as "pastic potted", "plastic plotted", or "pastic plotted") are deemed to involve the replacement of one syllabic constituent by another (e.g., replacement of /p/ by /pl/) rather as than as addition, deletion or shift errors. This allows these errors to be categorised as anticipations, perseverations, and exchanges in the same way that single phoneme errors would be. A sound error was classified as SA if all intended occurrences of the intruding constituent were after the target location, and as SP if all intended occurrences were before. An error was SE if there was a corresponding substitution in the same utterance (e.g., "pastic plotted...” for "plastic potted...” is SE). For errors in which the intruding constituent occurs both before and after the target, the error was classified as SAP if the before and after constituents were equidistant in words from the target. For example, "brad's burned ban buns" is SAP. If the two intruding constituents were not equidistant, the error was classified on the basis of the closest potential source. For example, "plastic plotted pansy plants" is SP, and "plastic potted plansy plants" is SA. 
4. Multiple errors. More than one error in an utterance creates some ambiguity of classification. The following heuristics were used to resolve ambiguity: (a) try to classify as many of the errors as possible as exchanges as opposed to anticipations and perseverations; (b) prefer exchanges that are closer together than further apart; and (c) try to account for the utterance in terms of the fewest error categories. For example, for “Pam’s plain plaid pan” prefer "Plam’s pain (SE) plaid plan (SP)” to “ Plam's (SA) pain (SP) plaid plan (SP)” or to “Plam’s (SA) pain plaid plan (distant SE).”

\section{Additional Rules}

The following were added after the first reliability check.

5. Onset bias. Assume that sound errors in a word's onset have a source in the onsets of nearby words. Hence, “Freida’s fabulous freaky fabric” spoken as “Freida’s fabulous freaky frabric" is SP, where the target onset /f/ in fabric is replaced by the onset /fr/ (from freaky and Frieda). This onset bias overrides a classification of SA from the nononset /r/ in fabric.

6. Look for exchanges first. Before determining whether errors are word or sound errors, look for possible exchanges. For example, for the phrase “tike’s tricky trike tire”, the error "trike's ticky trike tire” would be classified as “trike’s (WA) ticky (SP) trike tire" if the determination of whether an error is a word or sound is made before looking for exchanges. Instead, exchanges should be looked for first, resulting in classification in this case as SE (involving /t/ and /tr/).

\section{An Extended Example}

This complex case illustrates several classification principles (as well as the difficulty of classifying multiple errors). 
“Tike’s tricky trike tire” spoken as “trike’s tricky tike trire”.

The rule of looking for exchanges first finds two possibilities, the WE of trike and tike, and the SE of the onsets of tike and trire. These characterisations are contradictory because they each use the same error string tike, one analysis saying that it is the final part of a WE, and one saying that it is the initial part of an SE. If the WE option is chosen then the complete classification is “Trike’s tricky tike (WE) trire (SP).” (Note that trire is SP because of the onset bias.) If the SE option is chosen, the complete classification is “trike's (WA) tricky tike trire (SE).” Because both analyses have the same number of errors (2) and both follow the rule of looking for exchanges, the only basis for a tie breaker is the exchange distance. Hence, the WA-SE analysis was chosen. 


\section{Appendix C}

Tongue twisters used in Experiment 2

\section{Practice Examples}

Steve's stove smoulders smoke

Lisa’s lashes lack liner

goats grow good grass

Group A Tongue Twisters

Brad’s burned bran buns

chef's sooty shoe soles

Danny’s dripping dish drain

fine fresh free fish

five frantic fat frogs

Pam’s plain plaid pan

sappy shiny shop signs

Tike’s tricky trike tire

\section{Group B Tongue Twisters}

Bonnie’s brown bread box

brief beastly beach breezes

Floyd's fourth floor fort

Frieda’s fabulous freaky fabric

Gloria’s Greek green gloves 
plastic potted pansy plants

simple slender silver slippers

thirty three throbbing thumbs 


\section{Author Notes}

Janet I. Vousden, Department of Psychology, University of Warwick, Coventry, England; Elizabeth A. Maylor, Department of Psychology, University of Warwick, Coventry, England.

This research was presented as a poster at the $42^{\text {nd }}$ Annual Meeting of the Psychonomic Society, Orlando, USA, November 2001. It was supported by a grant from the Leverhulme Trust (F/215/AY). We are grateful to Brian Robinson, Jessica Hartley, and Kathryn Lovelock for assistance in data collection and error transcription, to Michelle Ellefson for helpful discussion, and to two anonymous reviewers for their comments and suggestions.

Correspondence concerning this paper should be addressed to Janet I. Vousden, Department of Psychology, University of Warwick, Coventry, CV4 7AL, England, or via electronic mail to J.I.Vousden@warwick.ac.uk. 


\section{Footnote}

${ }^{1}$ Note that the mean number of errors for the 16 participants on which this analysis is based was 12.13 for the slow rate $(S D=5.91)$, and 16.63 for the fast rate $(S D$ $=8.14)$. A paired $t$-test on the mean number of errors for these16 participants in the slow and fast condition revealed that significantly more errors were made at the fast rate, $t(15)$ $=-2.15, p<.05$, consistent with the findings from all 20 participants. 
Table 1

Example of an Error From Each Error Category For The Phrase "five frantic fat frogs"

\begin{tabular}{ll}
\hline \multicolumn{1}{c}{ Error Category } & \multicolumn{1}{c}{ Example } \\
\hline sound anticipation & frive frantic fat frogs \\
sound perseveration & five frantic fat fogs \\
sound exchange & frive fantic fat frogs \\
sound anticipation-perseveration & five frantic frat frogs \\
word anticipation & fat frantic fat frogs \\
word perseveration & $\underline{\text { five frantic five frogs }}$ \\
word exchange & five fat frantic frogs \\
word substitution & four frantic fat frogs \\
other & flive frantic fat frogs \\
\hline
\end{tabular}

Note: Errors are indicated in italics; the source of the error is underlined. 
Table 2

Mean Number of Errors and Mean AP for 8-Year-Olds, 11-Year-Olds and Adults in Experiment 1

\begin{tabular}{|c|c|c|c|c|c|c|}
\hline \multirow[b]{2}{*}{ Measure } & \multicolumn{2}{|c|}{$\begin{array}{l}\text { 8-Year-Olds } \\
\qquad(n=20)\end{array}$} & \multicolumn{2}{|c|}{$\begin{array}{c}\text { 11-Year-Olds } \\
(n=20)\end{array}$} & \multicolumn{2}{|c|}{$\begin{array}{l}\text { Adults } \\
(n=20)\end{array}$} \\
\hline & $M$ & $S D$ & $M$ & $S D$ & $M$ & $S D$ \\
\hline \multicolumn{7}{|l|}{ Overall } \\
\hline Errors & 54.35 & 15.95 & 23.15 & 7.09 & 12.80 & 7.76 \\
\hline AP & 0.42 & 0.13 & 0.45 & 0.19 & 0.61 & 0.29 \\
\hline \multicolumn{7}{|l|}{ Slow speech rate } \\
\hline Errors & 24.75 & 11.47 & 8.35 & 5.66 & 5.50 & 4.24 \\
\hline $\mathrm{AP}$ & 0.48 & 0.23 & $0.65^{\mathrm{a}}$ & 0.31 & $0.61^{\mathrm{b}}$ & - \\
\hline \multicolumn{7}{|l|}{ Fast speech rate } \\
\hline Errors & 29.60 & 10.18 & 14.80 & 6.24 & 7.30 & 5.30 \\
\hline AP & 0.37 & 0.18 & $0.38^{\mathrm{a}}$ & 0.26 & $0.51^{\mathrm{b}}$ & - \\
\hline
\end{tabular}

Note: AP is the anticipatory proportion of all anticipations and perseverations.

${ }^{\mathrm{a}} n=18 .{ }^{\mathrm{b}}$ AP calculated from group totals of anticipations and perseverations, not from the average of individual APs. 
Table 3

Background Data for Young and Older Participants in Experiment 2

\begin{tabular}{|c|c|c|c|c|c|}
\hline \multirow[b]{2}{*}{ Measure } & \multicolumn{2}{|c|}{$\begin{array}{l}\text { Young Adults } \\
\qquad(n=20)\end{array}$} & \multicolumn{2}{|c|}{$\begin{array}{l}\text { Older Adults } \\
\qquad(n=21)\end{array}$} & \multirow[b]{2}{*}{ Difference } \\
\hline & $M$ & $S D$ & $M$ & $S D$ & \\
\hline Current health $^{\mathrm{a}}$ & 4.40 & 0.68 & 3.95 & 0.67 & $t(39)=2.12^{b}$ \\
\hline Eyesight (corrected) $^{\mathrm{a}}$ & 4.60 & 0.50 & 4.05 & 0.38 & $t(39)=3.97^{\mathrm{b}}$ \\
\hline Hearing (corrected) ${ }^{\mathrm{a}}$ & 4.25 & 0.72 & 3.71 & 0.56 & $t(39)=2.67^{\mathrm{b}}$ \\
\hline Vocabulary $^{c}$ & 20.55 & 3.24 & 24.43 & 2.98 & $t(39)=-4.00^{b}$ \\
\hline Speech rate ${ }^{\mathrm{d}}$ & 3.86 & 0.62 & 6.54 & 1.80 & $t(39)=-6.32^{b}$ \\
\hline
\end{tabular}

${ }^{\text {a }}$ Self-rated on a 5-point scale $(1=$ very poor, $2=$ poor, $3=$ fair, $4=$ good, $5=$ very good $)$. ${ }^{\mathrm{b}} p<.05$ (two-tailed test). ${ }^{\mathrm{c}}$ Part 1 of the Mill Hill Vocabulary Test (Raven, Raven, \& Court, 1988); maximum score $=33 .{ }^{\mathrm{d}}$ Average time in seconds over two trials to count aloud from 1 to 20 as quickly as possible. 
Table 4

Mean Number of Errors and Mean AP for Young and Older Adults in Experiment 2

\begin{tabular}{|c|c|c|c|c|}
\hline \multirow[b]{2}{*}{ Measure } & \multicolumn{2}{|c|}{$\begin{array}{l}\text { Young Adults } \\
\qquad(n=20)\end{array}$} & \multicolumn{2}{|c|}{$\begin{array}{l}\text { Older Adults } \\
\qquad(n=21)\end{array}$} \\
\hline & $M$ & $S D$ & $M$ & $S D$ \\
\hline \multicolumn{5}{|l|}{ Slow speech rate } \\
\hline Errors & 10.10 & 6.78 & 11.95 & 6.16 \\
\hline AP & $0.53^{\mathrm{a}}$ & 0.26 & 0.56 & 0.29 \\
\hline \multicolumn{5}{|l|}{ Fast speech rate } \\
\hline Errors & 14.75 & 8.38 & - & - \\
\hline AP & $0.35^{\mathrm{a}}$ & 0.26 & - & - \\
\hline
\end{tabular}

Note: AP is the anticipatory proportion of errors.

${ }^{\mathrm{a}} n=16$ 


\section{Figure Caption}

Figure 1 . The anticipatory proportion (AP) as a function of $\log _{10}$ (Error Probability) for child and adult age groups from Experiment 1 (closed symbols), and young and older adults from Experiment 2 (open symbols). The labels S and F indicate data from the slow and fast speech rate conditions, respectively. 
Figure 1

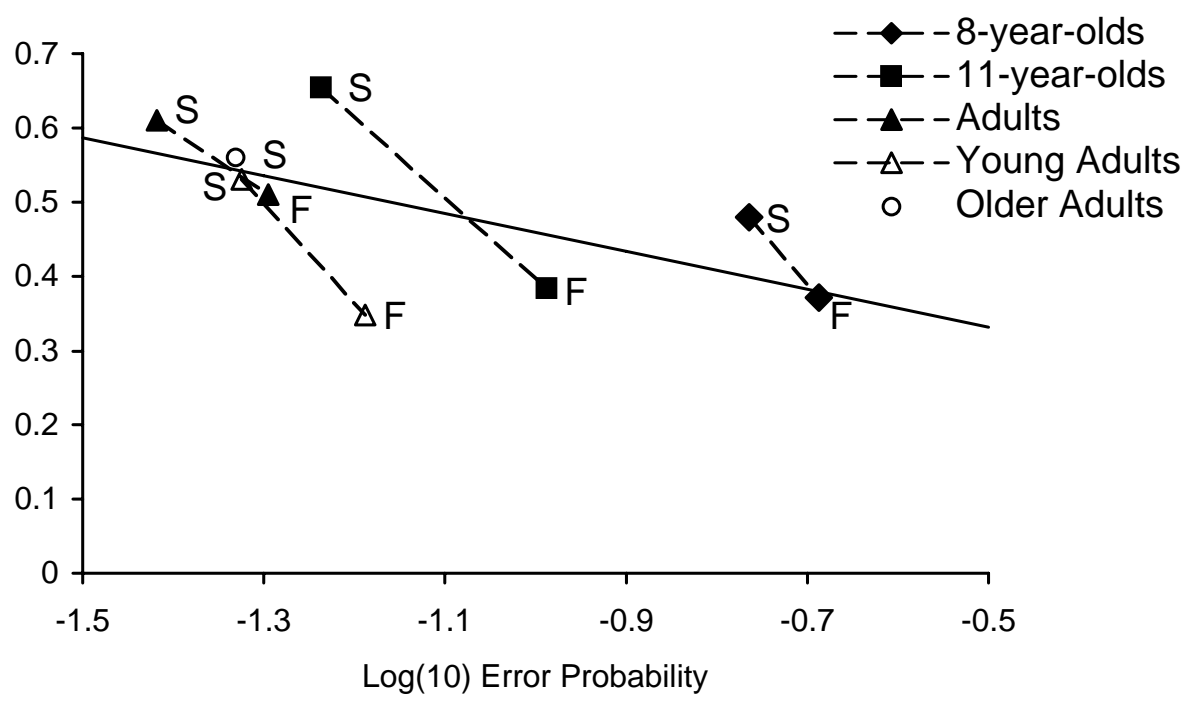


Speech errors 58 